\title{
Evaluación de los planes curriculares y sílabos de las asignaturas de la Facultad de Medicina de la UNMSM, sobre la atención integral de salud a personas afectadas por la violencia*
}

\author{
Assessment of Faculty of Medicine, Universidad Nacional Mayor de San Marcos curricula \\ and syllabi on subjects related to integral health care of persons affected by violence
}

\author{
Eva Miranda, Fausto Garmendia, Alberto Perales, Pedro Mendoza, Jorge Miano, Walter Calderón \\ Programa Permanente de Capacitación para la Atención Integral de las Víctimas de la Violencia, Facultad de Medicina, Universidad Nacional Mayor de San Marcos. Lima, Perú. \\ * Trabajo realizado con soporte de la Agencia de Cooperación Internacional del Japón (JICA) y de la Facultad de Medicina, UNMSM.
}

\begin{abstract}
Resumen
Propósito: En el año 2005 se realizó un Estudio de Línea de Base en la Facultad de Medicina de la Universidad Nacional Mayor de San Marcos (UNMSM) para conocer la realidad de la formación de los alumnos del pregrado sobre conocimientos y competencias relacionados a la violencia y violación de derechos humanos (DDHH), bajo la hipótesis que este problema nacional de salud no había sido considerado en forma adecuada y suficiente en los planes curriculares de la Facultad de Medicina. Objetivos: Diagnosticar el proceso de enseñanza-aprendizaje de la Facultad de Medicina sobre la atención integral de salud a personas afectadas por efectos de la violencia y violación de los derechos humanos. Diseño: Estudio descriptivo, transversal evaluativo de análisis. Institución: Facultad de Medicina, Universidad Nacional Mayor de San Marcos. Materiales: Planes de estudios de las 5 escuelas académico profesionales (EAP) de la Facultad. Intervenciones: Por juicio de un grupo de expertos, se seleccionó 81 asignaturas que, por sus características, debían contener temas relacionados con la violencia y violación de los derechos humanos (DDHH). Se analizó los sílabos de las asignaturas seleccionadas. Se efectuó coordinaciones con los directores de las 5 Escuelas Académico Profesionales. Se realizó presentaciones de los objetivos de la investigación y del Programa a los coordinadores de los departamentos de Psiquiatría, Medicina, Cirugía, Ginecología y Obstetricia, Pediatría, Medicina Preventiva y Salud Pública, así como entrevistas a docentes interesados en el tema de violencia y violación de los DDHH. A los docentes que acudieron a la entrevista se les indagó sobre su conocimiento, experiencia e interés sobre los temas señalados y se les explicó las características del Programa, con la finalidad de seleccionarlos para una futura capacitación. Principales medidas de resultados: Contenido sobre violencia en los sílabos de las escuelas académico profesionales. Resultados: El estudio demostró que solo 30/81 sílabos (37\%) tenía algún contenido sobre violencia, de los cuales correspondía a la EAP de Medicina 9/19 (47,3\%), EAP de Obstetricia 9/20 (45\%), EAP de Enfermería 5/12 (41,6\%), EAP de Tecnología Médica 6/19 (31,5\%) y la EAP de Nutrición 1/11 (9,1\%). Conclusiones: Estos resultados demuestraron la validez de la hipótesis, que efectivamente existía un vacío en la formación de los futuros profesionales de la salud respecto a sus conocimientos y competencias sobre violencia.

Palabras clave: Atención integral de salud; violencia; violaciones de los derechos humanos.
\end{abstract}

\begin{abstract}
Aims: In 2005 a baseline study was conducted in order to know the curricular plans and syllabi contents of the Faculty of Medicine, Universidad Nacional Mayor de San Marcos, related to violence health disorders given to undergraduate students of the 5 Academic Professional Schools (APS), under the hypothesis that this national health problem has not been considered sufficiently and adequately in the curricular plans of the Faculty. Objectives: To determine the Faculty of Medicine's teaching-learning process on integral health care to persons affected by violence and human rights violation. Design: Descriptive, transversal evaluative analysis. Setting: Faculty of Medicine, Universidad Nacional Mayor de San Marcos, Lima, Peru. Materials: Curricular plans of the 5 Faculty's academic professional schools. Interventions: A total of 81 syllabi courses were considered would have contents on themes related to violence. Coordination work with the directors of the Academic Professional Schools of Medicine, Midwifes, Nursery, Medical Technology and Nutrition was carried out. Formal presentations of the objectives of the Program and this research were done to the Department heads of Medicine, Surgery, Pediatrics, Gynecology-Obstetrics, Psychiatry, Preventive Medicine and Public Health. Interviews to professors to determine their knowledge, experience and interest in the field of violence were conducted as to consider future training. Main outcome measures: Academic professional schools' curricular plans contents on violence. Results: This study showed that only 30 out of 81 syllabi (37\%) had any content on violence, distributed as follows: Medicine $9 / 19$ (47,3\%), Midwifes 9/20 (45\%), Nursery 5/12 (41,6\%), Medical Technology $6 / 19(31,5 \%)$ and Nutrition $1 / 11(9,1 \%)$. Conclusions: These results demonstrate the validity of the work hypothesis in the sense that teaching of violence health consequences in the Faculty of Medicine had not been sufficiently considered.
\end{abstract}

Key words: Comprehensive health care; violence; human rights abuses.

\section{INTRODUCCIÓN}

En las tres últimas décadas, se ha generado evidencia científica sobre el efecto marcado de la violencia sobre la salud de las personas, que no solo indica mayores tasas de angustia, estrés postraumático y depresión y otros trastornos de salud física y mental, sino también reducción del ca- pital social, con marcados índices de desesperanza, desmoralización, disminución de la calidad de vida, migración forzada, reducción de la privacidad y desarrollo humanos ${ }^{(1)}$. En ese mismo tiempo, en el Perú se ha evidenciado, con llamativa intensidad, el desarrollo de diferentes formas de violencia y diversas violaciones de los derechos humanos que han originado severos problemas de salud ${ }^{(2)}$.

En el año 2003, la Comisión de la Verdad y Reconciliación presentó al Gobierno del Perú el informe final de sus investigaciones sobre la violencia política, desatada en el período de $1980 \mathrm{al}$ año 2000, en el que señaló la necesidad de 
efectuar reparaciones en diversos campos, entre ellos de la salud y educación. Hizo énfasis en la inexistencia de recursos humanos suficientemente capacitados para atender los problemas de salud de las víctimas de la violencia ${ }^{(3)}$.

Por ello, la Facultad de Medicina de la Universidad Nacional Mayor de San Marcos (UNMSM) creó, en ese mismo año, el Programa Permanente de Capacitación para la Atención Integral de las Víctimas de la Violencia (PPCAIVV) que, entre otras actividades, desarrolló un análisis colectivo a través de Talleres de Análisis de Involucrados y de Problemas (4), con la participación de la Agencia de Cooperación del Japón (JICA), docentes del Harvard Refugee Trauma Program (HRTP) de la Universidad de Harvard, autoridades políticas y de salud, miembros de la Comisión de la Verdad y Reconciliación (CVR), miembros del Programa de Apoyo al Repoblamiento, la sociedad civil, docentes universitarios y representantes de las víctimas de la violencia.

Una de las conclusiones de ese análisis fue la perentoriedad de evaluar la organización académica, planes curriculares, asignaturas y competencias de los estudiantes de la Facultad de Medicina, respecto a la atención integral de salud a pacientes traumatizados por la violencia y violación de los derechos humanos (DDHH), tendiente a optimizar los procesos académicos y efectuar cambios de los planes de estudios que permitieran la formación de profesionales de las ciencias de la salud competentes para atender en forma integral a las víctimas de la violencia.

Con tal propósito, se efectuó el Estudio de Línea de Base en la Facultad de Medicina de la UNMSM, motivo del presente trabajo, relacionado con la evaluación de los planes curriculares y asignaturas respecto a los contenidos sobre derechos humanos y atención primaria de salud a personas afectadas por la violencia, las necesidades de capacitación de los docentes para la implementación del proyecto y la evaluación de las competencias logradas por los estudiantes.

Bajo la hipótesis que este problema nacional de salud no había sido considerado en forma adecuada y suficiente en los planes curriculares de la Facultad de Medicina, se inició esta investigación. El objetivo general fue diagnosticar el proceso de enseñanza-aprendizaje de la Facultad de Medicina sobre la atención integral de salud a personas afectadas por efectos de la violencia y violación de los derechos humanos. Los objetivos específicos fueron evaluar los planes curriculares de las Escuelas participantes, en relación a la atención integral de salud por efectos de la violencia y violación de derechos humanos; evaluar los sílabos de las asignaturas clínicas y comunitarias, en relación a la atención integral de salud por efectos de la violencia y violación de derechos humanos; determinar las necesidades de capacitación de los docentes acerca del tema en estudio; y proponer modificaciones de los planes curriculares y de los sílabos de las asignaturas evaluadas

\section{MÉTODOS}

En el año 2005, se realizó un estudio descriptivo, transversal, sobre los planes de estudios de las Escuelas Académico Profesionales (EAP) de Medicina, Obstetricia, Enfermería, Tecnología Médica y Nutrición de la Facultad de Medicina de San Fernando de la Universidad Nacional Mayor de San Marcos. Un grupo de expertos, integrado por especialistas en salud mental, derechos humanos, medicina interna, salud pública, educación y gestión universitaria, seleccionaron un grupo de 81 asignaturas que por sus características, deberían contener temas relacionados con la violencia y derechos humanos.

Se analizaron los sílabos de las asignaturas seleccionadas, utilizando una ficha de revisión documental que contiene la estructura de los sílabos, aprobada por la Facultad de Medicina, con la finalidad de revisar en cada una y todas de sus partes la existencia o no de contenidos sobre violencia, víctimas, derechos humanos, derechos del paciente, violación, abuso, trauma, desastres, negligencia, abandono, ética, entre otros, para llenar los datos obtenidos del análisis. Figura 1.

Se coordinó con los directores de las 5 Escuelas Académico Profesionales de la Facultad. Se realizó presentaciones de los objetivos de la investigación y del Programa a los coordinadores de los departamentos de Psiquiatría, Medicina, Cirugía, Ginecología y Obstetricia, Pediatría, Medicina Preventiva y Salud Pública.

Se efectuó entrevistas semiestructuradas a docentes interesados en el tema de violencia y violación de los $\mathrm{DDHH}$, mediante requerimientos a los coordinadores de los departamentos académicos, por parte de un mínimo de 4 expertos. Se indagó sobre datos generales -como edad, condición docente, categoría, clase, departamento, escuela-, así como más específicos -como interés en el tema de la violencia, experiencia en el tema de violencia, estado de salud que permitiera replicar enseñanzas en lugares de altura y selva, grado de compromiso y tiempo disponible para continuar las actividades del Programa-, se estableció su conocimiento verbal del idioma inglés, conocimiento de idiomas nativos como el quechua y, además, se les explicó las características del proyecto, con la finalidad de seleccionarlos para una futura capacitación.

\section{RESULTADOS}

El 100\% de asignaturas tuvo un formato estándar en su presentación, de acuerdo a las Normas de Elaboración de Sílabos de la Facultad de Medicina. La mayoría de los sílabos no explicita contenidos relacionados con la atención integral de salud a víctimas de violencia y violación de derechos humanos (ver tabla 1).

Así, en la EAP de Medicina Humana solo 9 de 19 cursos (47,3\%) contenían alguna referencia relacionada a la violencia (abuso, trauma, desastre, violación, víctimas, derechos humanos del paciente y ética).

En la EAP de Obstetricia, 9 de 20 asignaturas $(45 \%)$ tenían una mención a algún aspecto relacionado a la violencia. Las asignaturas que la contenían eran del área de salud mental y ética; esta última se refería a temas de bioética, valores y derechos humanos y género. Las asignaturas que abordaban la atención integral de salud, centraban su desarrollo a la atención de los aspectos físicos y en menor proporción a los aspectos psicológicos, 
Figura 1. Modelo de intervención del PPCAIVV en la Facultad de Medicina.

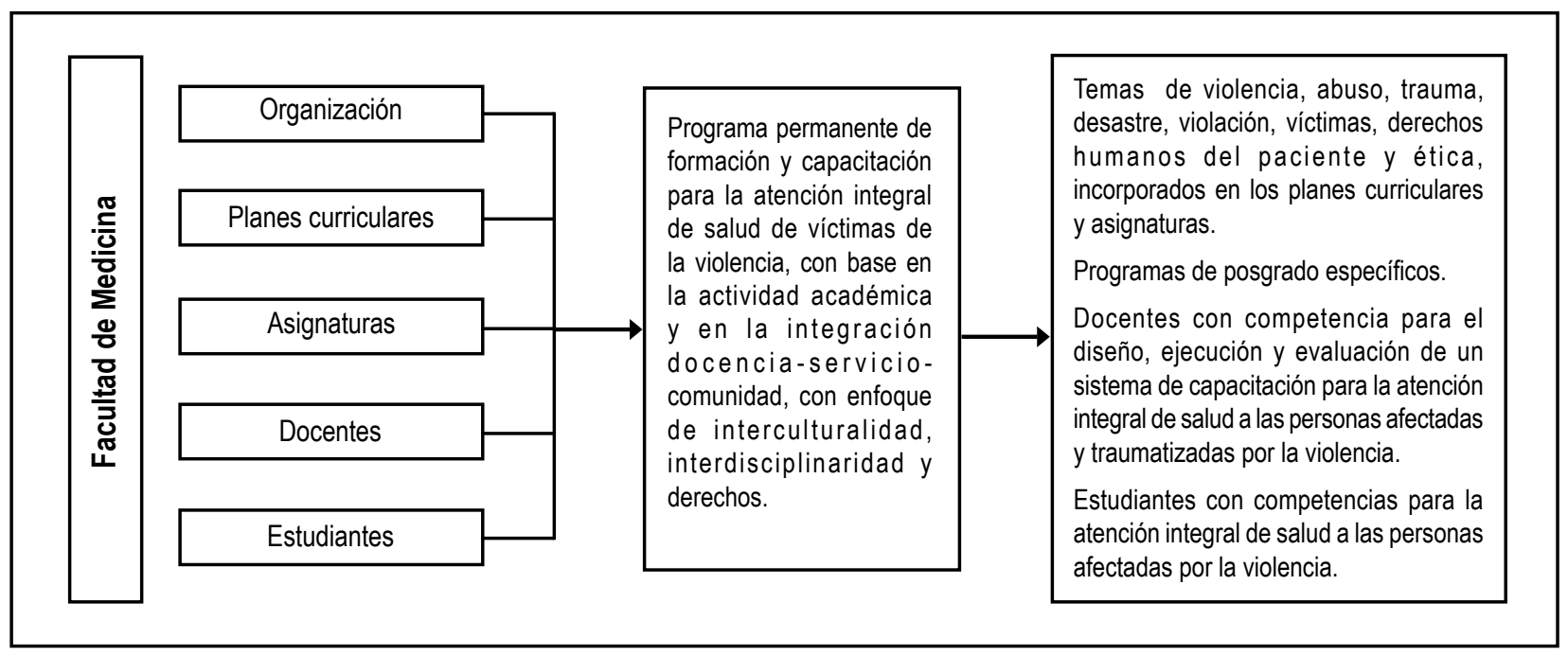

mucho menos a los sociales, lo espiritual, estaba casi ausente.

En la Escuela Académico Profesional de Enfermería, 5/12 asignaturas (40,6\%) tenían contenidos sobre violencia. Esta escuela es las que más ha desarrollado el tema de atención integral de salud, el trabajo con la comunidad y el enfoque intercultural. Sin embargo, existen algunos contenidos que profundizan aspectos médicos más que de enfermería. Hay ausencia de contenidos relacionados con la recuperación integral y reparación de las víctimas de violencia, conformación y articulación de redes sociales, consejería y apoyo emocional orientado hacia la con-

Tabla 1. Revisión documental de los 81 sílabos seleccionados.

\begin{tabular}{lccccc}
\hline \multicolumn{1}{c}{ Sección } & \multicolumn{5}{c}{ Resultados } \\
\cline { 2 - 6 } & Medicina & Obstetricia & Enfermería & $\begin{array}{c}\text { Tecnología } \\
\text { Médica }\end{array}$ & Nutrición \\
\hline $\mathrm{N}^{\circ}$ de sílabos & 19 & 20 & 12 & 19 & 11 \\
Sumilla & $21 \%$ & $5 \%$ & $50 \%$ & $11 \%$ & $100 \%$ \\
Competencias & $15 \%$ & $25 \%$ & $75 \%$ & $15,7 \%$ & $10 \%$ \\
Personal docente * & $100 \%$ & $95 \%$ & $75 \%$ & $74 \%$ & No se precisa \\
Metodología de enseñanza & - & $13,3 \%$ & $16,6 \%$ & - & $100 \%$ \\
Organización & - & - & - & - & $100 \%$ \\
Sistema de evaluación & - & $5 \%$ & - & - & $100 \%$ \\
Bibliografía & $26 \%$ & $5 \%$ & $43 \%$ & $10 \%$ & $20 \%$ \\
Instrucciones generales & - & $5 \%$ & - & - & $100 \%$ \\
Programa calendarizado & $9 / 19(47 \%)$ & $9 / 20(45 \%)$ & $5 / 12(40,6 \%)$ & $6 / 19(31,5 \%)$ & $1 / 11(9,1 \%)$ \\
Otros & \multicolumn{5}{c}{$\begin{array}{c}\text { Se dicta algunas } \\
\text { asignaturas en } \\
\text { Huaycán }\end{array}$} \\
\hline
\end{tabular}

${ }^{\text {* }}$ Porcentaje de docentes propios de la Facultad. ducta de búsqueda de ayuda y solución de problemas. Así mismo, son escasos los contenidos sobre la promoción de la salud mental con enfoque intercultural, para favorecer estilos de vida saludables.

En la EAP de Tecnología, 6 de 19 $(31,5 \%)$ asignaturas seleccionadas contenían temas sobre violencia. En la EAP de Nutrición, se halló que solo 1 de 11 $(9,1 \%)$ asignaturas había considerado aspectos de enseñanza sobre violencia.

Estos resultados demuestran la validez de la hipótesis de trabajo de la investigación realizada en el campo de la docencia médica, esto es, que la enseñanza sobre

\section{DISCUSIÓN}

Se debe considerar que la reforma curricular de las entidades educativas, en este caso de las facultades de medicina, no se debe circunscribir a seminarios curriculares, sino que debe ser un proceso permanente de análisis y cambios de acuerdo a las necesidades de atención de la comunidad.

Por las características de los problemas de salud que ocasionan las diversas formas de violencia, el proceso de enseñanzaaprendizaje se debe orientar a la atención integral de salud de las personas afectadas. En ese sentido, el PPCAIVV de la Facultad de Medicina de UNMSM propicia que la atención integral de salud es la atención segura, oportuna, continua, humana, con enfoque intercultural -es decir, con calidad- de los aspectos físicos, psicológicos, sociales y 
espirituales de la persona afectada por efectos de la violencia y violación de los derechos humanos y está orientada a la promoción, prevención, recuperación y rehabilitación de la salud en el contexto de su familia y comunidad, con actitud ética, compromiso social y moral y el mayor respeto por la vida humana. En consecuencia, la recuperación integral de la persona incluye las dimensiones psicológica, familiar, física, laboral y de participación social, tomando en cuenta las diferencias culturales y las necesidades de las víctimas. Para ello, se requiere de profesionales, de formación multidisciplinaria, que trabajen en equipo y participen en la reconstitución de las redes de soporte social y en el fortalecimiento de las capacidades para el desarrollo personal y social y así contribuir a favorecer el desarrollo de la autonomía necesaria en las víctimas, para reconstruir su proyecto de vida, individual y colectivo ${ }^{(6)}$.

La presente investigación permite aseverar que existe una necesidad perentoria de evaluar la organización académica, planes curriculares, asignaturas y competencias de los estudiantes de pre y post grado de la Facultad de Medicina, respecto a la atención integral de salud a pacientes traumatizados por la violencia y violación de los derechos humanos y así mejorar los procesos de enseñanza-aprendizaje, para lo cual se ha demostrado la validez y eficacia que permiten los estudios de línea de base como instrumentos de investigación en el campo de la docencia, para alcanzar dichos fines ${ }^{(7,8)}$.

Si bien es cierto que, desde el año 2005, gracias a las coordinaciones que el PPCAIVV ha efectuado con los directores de las escuelas y de los departamentos académicos de la Facultad de Medicina, ya se ha obtenido cambios curriculares sobre la enseñanza de diversos aspectos de la violencia, es conveniente se tomen en consideración las siguientes recomendaciones preliminares:

1. Continuar con la Reforma Curricular, incorporando los ejes integradores aprobados: la ética, la salud comunitaria y la investigación.
2. Incluir en los sílabos, en forma explícita, el tema de la atención integral de salud a víctimas de violencia y violación de derechos humanos. El tema debe ser incorporado en diferentes grados, de acuerdo y a la naturaleza de las asignaturas, tales como geografía de la violencia en el Perú, historia de la violencia en el Perú, epidemiología de la violencia, diagnóstico precoz de problemas psiquiátricos y de salud mental del niño, adolescente, adulto y adulto mayor, generados por la violencia (trauma agudo y crónico, depresión, trastorno de estrés postraumático, alcoholismo, angustia). Tratamiento farmacológico y psicoterapéutico en sus diversas modalidades -individual, grupal y familiar. Referencia oportuna y contrarreferencia. Recuperación integral de las víctimas de violencia, con apoyo de la comunidad. Formación y articulación de redes sociales. Seguimiento del cuidado de las víctimas de la violencia hasta su recuperación. Programas de reconciliación y reparación de víctimas de la violencia y violación de derechos humanos. Consejería y apoyo emocional, orientando a la víctima de violencia y a su familia hacia la conducta de búsqueda de ayuda y solución de problemas. Manejo del dolor físico y psicológico y el proceso de duelo de la víctima de violencia, la familia y comunidad. Manejo de instrumentos, mecanismos y relaciones para establecer un abordaje espiritual en la terapia de las víctimas, la familia y la comunidad, tomando en cuenta los factores culturales. Prevención de los trastornos mentales y secuelas relacionadas con la violencia. Promoción de la salud mental con enfoque intercultural, favoreciendo estilos de vida saludables. Entrenamiento en procesos de resiliencia y autocuidado.

3. Establecer mecanismos de seguimiento del desarrollo de los contenidos.

4. Evaluar la organización académica, planes curriculares y competencias de los estudiantes de pre y post grado de la Facultad de Medicina, respecto a la atención integral de salud a pacientes traumatizados por la violencia y violación de los derechos humanos.

\section{REFERENCIAS BIBLIOGRÁFICAS}

1. OPS-OMS. Informe Mundial sobre Violencia y Salud. Washington: OPS/OMS; 2002.

2. Viviano T. Maltrato en el hogar a las personas adultas mayores: Estadísticas para la reflexión y pautas para promover el buen trato. Lima: Ministerio de la Mujer y Desarrollo Social; 2008. Disponible en: http://www.mimdes.gob.pe/files/ SERVICIOS/PNCVFS/2009_maltrato_hogar_ personas_mayores.pdf

3. Comisión de la Verdad y Reconciliación. Informe Final. Lima; CVR; 2003.

4. Garmendia F, Perales A, Miano J, Miranda E, Watanabe E. Derechos humanos y atención primaria en salud a personas traumatizadas por la violencia en el Perú - Talleres de Análisis de Involucrados y de Problemas. Cusco, Lima e Ica. (documento interno). Lima; 2003.

5. Miranda E, Garmendia F, Perales A, Mendoza P, Miano J, Calderón W. Evaluación de competencias de los internos de medicina para la atención integral de salud a personas afectadas por la violencia. Propuesta de mejoras-UNMSM - 2005. II Congreso Peruano de Educación Médica. Trujillo, Perú, 5-8 diciembre 2007.

6. Ministerio de Salud. Modelo de atención integral en salud (MAIS). Lima: Minsa; 2003.

7. Garmendia F, Perales A, Miranda E, Mendoza P, Calderón W, Miano J, Cano B. Estudios de línea de base como metodología para una mejor capacitación para la atención integral de las víctimas de violencia. Libro de Resúmenes. I Congreso Peruano de Educación Médica, Lima, Perú. 30-nov a 3 dic, 2005. p. 20.

8. PPCAIPP. Informe Global de los Estudios de Línea de Base, Facultad de Medicina, UNMSM. Lima: UNMSM; 2005.

Manuscrito recibido el 12 de junio de 2009 y aceptado para publicación el 9 de setiembre de 2009.

Correspondencia:

Dr. Fausto Garmendia Lorena

Instituto de Investigaciones Clínicas

Facultad de Medicina, UNMSM

Hospital Nacional Dos de Mayo

Parque de la Medicina s/n. Av Grau Cdra. 13

Lima 1, Perú

Correo-e: faustogarmendia@yahoo.com 